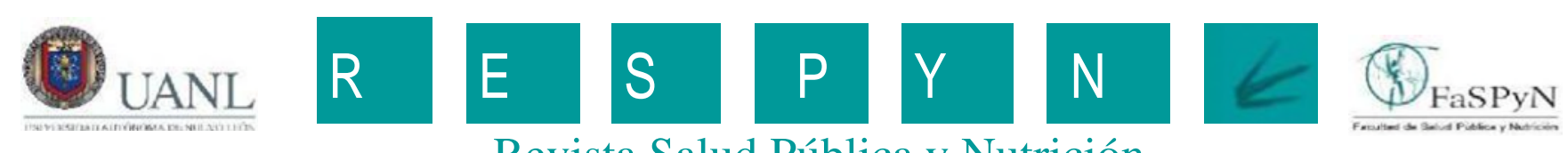

Revista Salud Pública y Nutrición

\title{
DETECCIÓN PRECOZ DEL CÁNCER EN LA MUJER, ENORME DESAFÍO DE SALUD EN MÉXICO. ALGUNAS REFLEXIONES
}

EARLY DETECTION OF CANCER IN WOMEN, A HUGE HEALTH CHALLENGE IN MEXICO. SOME REFLECTIONS

\section{Dórame-López Norma Alicia1, Tejada-Tayabas Luz María², Galarza-Tejada Dulce María³, Moreno- González María Mercedes ${ }^{4}$}

1 Universidad de Sonora Fac. Enfermería. 2 Benemérita Universidad Autónoma de San Luis Potosí y Universidad Michoacana de San Nicolás de Hidalgo. 3 Benemérita Universidad Autónoma de San Luis Potosí. 4 Universidad de Guanajuato. Campus Celaya Salvatierra

\footnotetext{
Citation: Dórame-López NA., Tejada-Tayabas LM., Galarza-Tejada DM., Moreno-González MM. (2017) Detección precoz del cáncer en la mujer, enorme desafío de salud en México. Algunas reflexiones, Revista de Salud Pública y Nutrición, 16(3), 14 - 22

Editor: Esteban G. Ramos Peña, Dr. CS., Universidad Autónoma de Nuevo león, Facultad de Salud Pública, Monterrey Nuevo León, México.

Copyright: @2017 Dórame-López NA. This is an open-access article distributed under the terms of Creative Commons Attribution License [CC BYND 4.0], which permits unrestricted use, distribution, and reproduction in any medium, provided the original author and source are credited.

Competing interests: The authors have declared that no competing interests exist.

DOI: https://doi.org/10.29105/respyn16.3-3

*Email: Imtayabas@yahoo.com.mx
} 


\title{
DETECCIÓN PRECOZ DEL CÁNCER EN LA MUJER, ENORME DESAFÍO DE SALUD EN MÉXICO. ALGUNAS REFLEXIONES
}

\author{
Dórame-López Norma Alicia1, Tejada-Tayabas Luz María², Galarza-Tejada Dulce María ${ }^{3}$, Moreno-González \\ María Mercedes ${ }^{4}$
}

\author{
1 Universidad de Sonora Fac. Enfermería. 2 Benemérita Universidad Autónoma de San Luis Potosí y \\ Universidad Michoacana de San Nicolás de Hidalgo. 3 Benemérita Universidad Autónoma de San Luis \\ Potosí. 4 Universidad de Guanajuato. Campus Celaya Salvatierra
}

\begin{abstract}
RESUMEN
Introducción: El incremento acelerado en la prevalencia del cáncer de mama (CaMa) y cáncer cervicouterino (CaCu) así como su elevada mortalidad en México, representan un gran desafío para el sistema de salud mexicano. A pesar de los esfuerzos y las acciones emprendidas para reducir las cifras, el impacto no ha sido el esperado. La evidencia establece que si el cáncer se detecta en estadíos iniciales es curable, tiene un mejor pronóstico, mayores tasas de supervivencia, los tratamientos son más asequibles y pueden administrarse con menos recursos y costos. Por ello la importancia de la prevención y detección temprana de la enfermedad y su tratamiento oportuno, lo cual requiere de una educación para generar conciencia y no solo proporcionar información a las mujeres, así como de garantizar el fácil acceso a servicios de salud de calidad en atención primaria. El objetivo de este ensayo es presentar una reflexión crítica sobre algunas de las razones por las que la detección precoz del cáncer en la mujer se mantiene como uno de los principales desafíos de salud en México. Conclusiones: Para ello se plantean algunas ideas reflexivas sobre: el soporte de las políticas públicas a las acciones de atención primaria, la relevancia del nivel de conocimientos en las mujeres respecto a la prevención y detección oportuna de CaMa y $\mathrm{CaCu}$

Palabras clave: Promoción de la salud, detección precoz de cáncer, neoplasia de la mama, neoplasia de cuello del útero
\end{abstract}

\section{ABSTRACT}

The rapid increased the prevalence of breast cancer $(B C)$ and cervical cancer $(\mathrm{CC})$ and its high mortality in Mexico represent a major challenge for the Mexican health system. Despite the efforts and actions taken to reduce the numbers, the impact has not been as expected. The evidence establishes that if the cancer is detected in early stages is curable, has a better prognosis, higher survival rates, and the treatments are more affordable and can be managed with fewer resources and costs. Hence it's important the prevention and early detection of disease and timely treatment, which requires education to develop awareness not only provide information to women, and it is important to ensure easy access to health services quality primary care. The aim of this paper is to present a critical reflection about some of the reasons why early detection of cancer in women is maintained as one of the major health challenges in Mexico. To this end, some reflective ideas are put forward about: the support of public policies for primary care actions, the relevance of the level of knowledge in women regarding the prevention and timely detection of $\mathrm{CaMa}$ and $\mathrm{CaCu}$.

Key words: Health promotion, early detection of cancer, breast Cancer, neck of the uterus neoplasm. 


\section{INTRODUCCIÓN}

El cáncer (Ca) en la mujer representa uno de los principales problemas de salud pública a nivel mundial, específicamente el Ca de mama (CaMa) y el $\mathrm{Ca}$ cervicouterino $(\mathrm{CaCu})$ ocupan las primeras causas de mortalidad femenina por tumores malignos, tanto en los países de ingresos altos como de ingresos medianos y bajos (Organización Mundial de la Salud, 2013). México no es la excepción, puesto que ambos tipos de $\mathrm{Ca}$ son problemas prioritarios dentro de la agenda de salud nacional.

En este país, el CaMa presentó una tendencia al incremento, la tasa de mortalidad tuvo una transición de 11.7/100 000 mujeres fallecidas en 1980 a una tasa de 17/100 000 en 2009 (Knaul \& Hornelas, 2014). Pasó de ser la causa número 15 de defunción en 1980 a ser la segunda en 2012 (World Health Organization, 2012). Así mismo, durante el período 2007 a 2014 la incidencia de CaMa se mantuvo en ascenso, en 2014 se reportó la cifra más alta con 29 casos nuevos por cada 100 mil mujeres de 20 años y más, el $90 \%$ de los cuales fueron detectados en las etapas III y IV (INEGI, 2014). Tales cifras permiten proyectar que para el 2025 habrá un incremento importante en la incidencia y mortalidad con 30,993 casos nuevos y 9,013 fallecimientos (Secretaría de Salud, 2015).

Por su parte, el $\mathrm{CaCu}$ continúa como una de las principales causas de mortalidad femenina, con una tasa de 12 muertes por cada 100 mil mujeres de 25 años o más. Aunque ha venido mostrando una tendencia decreciente, mantiene índices altos en algunos estados del sur del país (Sánchez, 2012). De acuerdo con la OMS (2012), la frecuencia de casos nuevos de $\mathrm{CaCu}$ fue de 10,186 lo cual representa una tasa estandarizada a nivel mundial de 19.2 por 100 mil mujeres. Estos datos ubican a este tipo de $\mathrm{Ca}$ en el segundo lugar en frecuencia de morbilidad y mortalidad por neoplasias malignas en la mujer, después del CaMa (Siegel, Nishadham \& Jemal, 2012).

Muchas de las mujeres afectadas por el $\mathrm{CaCu}$ viven en un contexto socioeconómico y cultural de bajo estrato (Smith et al., 2015). En los países con menor desarrollo, la tasa es de 20 muertes por cada 100 mil mujeres, la cual se acentúa al compararla con la de los países desarrollados cuya tasa es de 2 muertes por cada 100 mil mujeres, la disminución en la tasa referida en tales países está vinculada con la realización del tamizaje, los diagnósticos certeros y los tratamientos oportunos (World Health Organization, 2015).

A pesar de los esfuerzos emprendidos por el sistema de salud mexicano, la detección tardía del $\mathrm{Ca}$ en la mujer es común, un $70.2 \%$ de los casos registrados del 2003 al 2008 se detectaron en estadios avanzados (López, Torres, Blanco, Hernández \& Knaul, 2014). En ello influye principalmente la demanda tardía de los estudios diagnósticos por parte de las mujeres, los retrasos en la atención para una detección oportuna y para el diagnóstico definitivo (Tejada, Amell \& Monárrez, 2015). Situación que no sólo provoca una disminución en la sobrevida de las mujeres, sino que conlleva el uso de procedimientos terapéuticos más agresivos y costosos que impactan a la mujer, a su familia y a la sociedad de una manera más contundente (Chávarri-Guerra et al., 2012).

La evidencia establece que si el $\mathrm{Ca}$ en la mujer $(\mathrm{CaMa}$ y $\mathrm{CACu})$ se detecta en estadios iniciales es curable, tiene un mejor pronóstico, mayores tasas de supervivencia, los tratamientos son más asequibles y pueden administrarse con menos recursos, tanto por el sistema de salud como por las familias afectadas (Knaul et al., 2012). Por ello la importancia de la prevención y detección temprana de la enfermedad y su tratamiento oportuno.

Este ensayo presenta una reflexión crítica sobre las razones por las que la detección precoz del $\mathrm{Ca}$ en la mujer se mantiene como uno de los principales desafíos de salud en México. Para ello se discute sobre el papel de las políticas públicas para sustentar las acciones de atención primaria. Se presentan también algunos datos de estudios realizados en mujeres mexicanas que permiten dar cuenta de la relevancia que tiene el conocimiento consciente sobre la prevención y detección oportuna de CaMa y $\mathrm{CaCu}$. Y la necesidad apremiante de fortalecer la educación para la salud y la consejería, para poder trazar actuaciones más efectivas de las usuarias en la reducción de los factores de riesgo que son modificables, e incrementar la demanda de la detección de estas patologías con la regularidad y oportunidad que se requiere. 


\section{LAS POLÍTICAS PÚBLICAS DIRIGIDAS A LA PREVENCIÓN Y DETECCIÓN OPORTUNA DE CAMA Y CACU EN MÉXICO.}

Las acciones para la prevención, detección y diagnóstico oportuno para estos tipos de cáncer en la mujer, son prioritarias en el sistema de salud mexicano y se sustentan en normas y políticas públicas específicas. El Plan Nacional de Desarrollo 2013-2018 (2013) establece de manera prioritaria que las instituciones del Sistema Nacional de Salud y la sociedad en su conjunto, "deben hacer de las acciones de protección, promoción y prevención, un eje prioritario para el mejoramiento de la salud, con el propósito de alcanzar la meta Nacional de un México incluyente". Así mismo, se describe en la presentación del Programa de Acción Específico para la Prevención y Control de Cáncer en la Mujer (PAEPCCM) (Secretaría de Salud PAEPCCM, 2015), la importancia de la prevención primaria y secundaria así como la prioridad de las actividades en el primer nivel de atención como impulsoras del cambio en el panorama epidemiológico del $\mathrm{Ca}$ en la mujer.

En el PAEPCCM se propone la prevención en tres niveles diferentes que se ejecutan según la población específica a que van dirigidas, las primeras acciones son educativas: encaminadas a toda la población, las segundas dirigidas a las mujeres por grupo de edad y las terceras para mujeres que tienen alto riesgo, con resultados anormales o sospecha clínica de cáncer (Secretaría de Salud PAEPCCM, 2015). Para cumplir con las líneas de acción, se proponen varias actividades, sin embargo, se aprecia que las de prevención y educación son mínimas, y las dirigidas al tamizaje son mayores y prioritarias. Ello puede considerarse congruente si se asume que el cribado mediante las técnicas correspondientes para cada tipo de cáncer, es la estrategia idónea para una detección precoz, no obstante, las acciones educativas son esenciales, y resultan ser la clave para que las mujeres de manera consciente se acerquen al tamizaje. Esta pudiera ser la principal razón por la cual las mujeres se acercan a los servicios para recibir un diagnóstico definitivo, solo cuando ellas perciben algunos síntomas y el cáncer se encuentra en estadios avanzados, más no para prevenirlo y procurar una detección temprana.

En lo concerniente a la detección oportuna del CaMa, la Norma Oficial Mexicana para la prevención, diagnóstico, tratamiento, control y vigilancia del CaMa (2011), establece tres estrategias: a) autoexploración mamaria (AE) a partir de los 20 años; b) exploración clínica (EC) practicada por personal capacitado a las mujeres a partir de los 25 años y; c) la realización regular de la mastografía (MA) bienal para todas las mujeres asintomáticas de 40 a 69 años.

Entre las tácticas citadas, la $\mathrm{AE}$ resulta el pilar fundamental de los programas de prevención y control. No obstante la sencillez de este examen, la $\mathrm{AE}$ tiene poca prevalencia entre las mujeres, pues se reporta que sólo una mujer de cada tres la llevan a cabo de manera efectiva (Cordova \& González, 2015).

Por su parte, la Norma Oficial Mexicana para la prevención, detección, diagnóstico, tratamiento, control y vigilancia epidemiológica del $\mathrm{CaCu}$ (2007), instituye que el personal de salud (médicos, enfermeras y técnicos capacitados) propondrá la prueba de detección de $\mathrm{CaCu}$ (Papanicolaou), a toda mujer de 25 a 64 años de edad, especialmente a las que refieren factores de riesgo y se le realizará a quien la solicite indistintamente de su edad. Asimismo indica que a las mujeres que obtengan resultados favorables en dos pruebas anuales sucesivas, esta se les realizará periódicamente cada tres años.

La citología vaginal o mejor conocida como Papanicolaou (Pap) es una prueba de tamizaje para detectar lesiones en el cérvix (Urdaneta et al., 2013). El estudio es de bajo costo, sencillo e indoloro, y el procedimiento dura menos de cinco minutos (Programa de acción específico para $\mathrm{CaCu}, 2008$ ) lo que facilita emplearlo en poblaciones extendidas. No obstante, la detección oportuna de $\mathrm{CaCu}$, tiene una cobertura desigual de la población femenina mayor de 25 años en las zonas rurales y en las urbanas, lo que incrementa el riesgo de morir a causa del $\mathrm{CaCu}$ en las poblaciones alejadas (Sánchez, 2012). Principalmente en lugares donde convergen factores como el bajo nivel educativo, y económico, además de algunos patrones culturales que impiden a las mujeres acercarse al cribado.

Según algunas investigaciones, (Aranguren-Pulido et al., 2017; Knaul et al., 2012), las dificultades de acceso a los servicios de salud es una de las razones 
que propician una pobre participación de las mujeres en programas de detección oportuna de $\mathrm{CaCu}$.

Las ventajas, facilidades y beneficios de la prueba son indiscutibles, no obstante, entre las principales barreras de su cobertura están también las relacionadas con aspectos culturales y cognitivos de las mujeres (Ehrenzweig, Marván \& Acosta, 2013).

\section{LOS CONOCIMIENTOS Y CONCIENCIA DE LAS MUJERES SOBRE EL CAMA Y LA DETECCIÓN OPORTUNA.}

La percepción real de riesgo conlleva un conjunto de conocimientos generales sobre la enfermedad, su evolución e impacto, sus factores asociados y las formas de prevención y detección oportuna. Diversos estudios muestran hallazgos sobre el nivel de conocimientos que poseen grupos específicos de mujeres mexicanas, respecto a los tipos de $\mathrm{Ca}$ a los que están expuestas, así como a las formas como pueden prevenir y detectar oportunamente la enfermedad. Datos que permiten reflexionar sobre el desafío de la educación a las mujeres para promover una detección más eficaz

Algunos estudios como el de Pérez, Romero, Benítez y Sandoval (2011), identificaron que de las mujeres participantes en el estudio, el grupo de mayor edad (47-57 años) y con menor grado de escolaridad cuenta con un menor nivel de conocimientos sobre el $\mathrm{CaMa}$, lo cual resulta relevante si se tiene en cuenta que este grupo de edad presenta mayor riesgo de padecer la enfermedad. Por su parte Campero y colaboradores (2014), en su exploración en comunidades con población indígena, identificaron que las mujeres poseen una noción vaga o errónea sobre el riesgo de padecer CaMa; el estudio expone que la mayoría de las mujeres objeto de estudio consideran que las jóvenes no son candidatas para padecer esta enfermedad, ni corren peligro alguno de desarrollarla sino hasta después de los 45 años. Este hallazgo refiere la falsa percepción de riesgo que presentan muchas mujeres.

Respecto a la AE mamaria, varios estudios (Gallegos, Zonana, Gonzáles \& Zapién, 2014; Jacobo-Galindo et al., 2014); López-Guevara et al., 2014), muestran una relación significativa entre la edad, el nivel de escolaridad y socieconómico de las mujeres con los conocimientos sobre la técnica de AE y su práctica regular. Específicamente los trabajos de López, Torres, Blanco, Hernández \& Knaul, (2014) y Yépez, Rosa, Guerrero 6 Gómez (2012) evidencían que una mayoría de las mujeres participantes en las investigaciones mostraron tener información sobre la importancia de realizar la AE, no obstante, pocas mujeres dominan la técnica correcta de la AE y la practican regularmente. Por su parte, Córdova, González \& Zavala, (2015) encontraron que la prevalencia de utilización correcta de la $\mathrm{AE}$ fue de apenas un $11 \%$. Ello muestra que la calidad de la técnica de la AE entre las mujeres no es la apropiada para una detección eficaz. La realidad es que una mayoría de las mujeres no práctica la $\mathrm{AE}$ de manera regular y los motivos exteriorizados para no realizarla se relacionaron con: tener miedo a detectar algo "extraño" (50\%), la desidia y la flojera (50\%) (Yépez et al., 2012) o bien por desconocimiento del procedimiento (17.6\%) (Sánchez et al., 2016).

Otro desafío mayor es que las mujeres acudan a la exploración clínica por personal calificado o a realizarse una mastografía. De acuerdo con la Encuesta Nacional de Salud y Nutrición 2012 (Gutierrez, Rivera, Shama, Oropeza \& Ávila, 2012) sólo un $36.3 \%$ de las mujeres de 20 años o más acudió a exploración clínica de senos (EC) en el año previo a la encuesta).

En el caso de la detección de CaMa a través de medios de diagnóstico, tan sólo un $15 \%$ de las mujeres de 40 a 49 años y un $26 \%$ de 50 a 69 años acudió a realizarse una mastografía. Según lo reportado por Campero y colaboradores (2014). Las mujeres de comunidades indígenas reconocieron la importancia de asistir al centro de salud una vez por año para que los proveedores de atención les realicen el EC, no obstante, no acuden como corresponde.

Según otros estudios (Agudelo, 2013; López et al., 2014; Jacobo-Galindo et al., 2014) la asiduidad de las mujeres al EC está asociada al nivel de escolaridad y a la edad, incrementándose en el grupo de más de 50 años, y en general, solo cuatro de cada 10 mujeres acuden a dicho examen anualmente.

Los principales motivos referidos por las usuarias para no realizarse el EC fueron que nunca se les ofreció por parte del personal de salud (83\%), por temor a que les encontraran un tumor $(9 \%)$ (López et al., 2014) o bien, porque su percepción ubica a la 
prueba como un procedimiento recomendable solo para mujeres mayores e ignoran la regularidad con que es recomendable realizarse el procedimiento (Campero et al., 2014).

Estos datos concuerdan con lo identificado por López y colaboradores (2014), respecto a los motivos de las mujeres entre 40 y 49 años para solicitar el estudio, entre los que destacan; haber detectado de manera incidental una "bolita" en la mama o presentar secreción del pezón (41.5\%), signos que corresponden al CaMa en evolución. El principal motivo de las mujeres de 50 años o más fue la edad $(69.7 \%)$ al considerarse a si mismas en mayor riesgo.

Los datos referidos muestran la necesidad de incrementar la educación para sensibilizar y concientizar a las mujeres en riesgo, sobre el CaMa y las alternativas para una detección precoz, e incluir a sus parejas o familiares cercanos. Particularmente procurar que el contenido de la información este acorde al contexto, bagaje cultural y escolar de las mujeres, a fin de sensibilizarlas sobre su riesgo y estrategias de protección. Es indispensable que las mujeres se familiaricen con los signos de alarma del CaMa y las consecuencias, en caso de no acudir tempranamente a los servicios para obtener un diagnóstico definitivo.

\section{CONOCIMIENTOS Y CONCIENCIA DE LAS MUJERES SOBRE EL CACU Y SU DETECCIÓN OPORTUNA}

$\mathrm{Al}$ igual que en el CaMa la detección precoz del $\mathrm{CaCu}$ depende particularmente del acercamiento de las mujeres al tamizaje y de la garantía de un fácil acceso a los servicios de atención a la salud.

Al respecto, Vargas, Vargas \& Tovar (2015), Llanes, Torres, Gómez \& Ochoa (2011), coinciden en que las acciones preventivas son fundamentales para su diagnóstico oportuno y tratamiento apropiado, siendo el Papanicolaou la prueba por elección, sin embargo, puntualizan que en la población mexicana no existe una cultura de prevención debido a diversos factores, por ejemplo que las mujeres tomen la decisión de no realizarse la prueba ya sea por estar condicionadas por sus parejas a no hacerlo, un limitado conocimiento y conciencia de riesgo o por la falta de acceso a los servicios de salud.
Campero y colaboradores (2014) dilucidó que las mujeres saben que acudir a realizarse el Pap permite detectar a tiempo alguna lesión y evitar el $\mathrm{CaCu}$, no obstante no lo practican con la regularidad requerida. Por su parte Ehrenzweig y colaboradores (2013), expone que todas las mujeres que conformaron el estudio sabían poco sobre los factores de riesgo del $\mathrm{CaCu}$, incluso aquellas con educación a nivel superior.

Entre los obstáculos para adherirse al tamizaje, en primer lugar se encontró el desconocimiento respecto a la edad requerida, seguido por el déficit de conocimiento sobre la frecuencia con que debe efectuarse el Pap y por último, los largos tiempos de espera en las unidades de salud.

\section{REFLEXIONES FINALES}

En términos generales, las mujeres poseen nociones sobre los tipos de cáncer a las que están expuestas y las estrategias para su detección oportuna. No obstante, se aprecian imprecisiones en la información respecto a los factores de riesgo, la técnica adecuada de AE, la edad a que deben iniciar las detecciones, así como la periodicidad del tamizaje para cada tipo de Ca. Evidentemente las mujeres cuentan con información, pero no logran integrarla a su contexto particular para construir una visión clara de su propio riesgo y las alternativas que tienen para reducirlo. Dado que el nivel de escolaridad y socioeconómico parece ser un determinante del apego correcto de las mujeres a los procedimientos de detección. Estos hallazgos exponen la relevancia de considerar las características sociales y culturales de las mujeres a fin de adaptar la educación y consejería a sus rasgos y proveerles no solo información, sino procurar el desarrollo de una conciencia de riesgo y de la necesidad del cuidado de la salud.

Queda en claro la relevancia de que las mujeres, por decisión propia, demanden la detección de CaMa y $\mathrm{CaCu}$ con la regularidad correspondiente y procuren evitar en lo posible los factores de riesgo asociados, así como la necesidad de recibir una educación para que adquieran conciencia de ello.

Por ende, es de esperarse que la población femenina con mayor grado de conocimientos respecto al CaMa y $\mathrm{CaCu}$, integrados a su esquema cognitivo y experiencias de vida cotidiana, llevará a cabo prácticas de prevención y detección oportuna más 
asiduamente, lo cual podría contribuir a acortar los índices de morbimortalidad y reducir el gasto en salud.

El sistema de salud hace esfuerzos por controlar y reducir el avance acelerado del cáncer en la mujer, las acciones de prevención y detección se califican como prioritarias de la atención en el primer nivel.

Sin embargo, parece que las estrategias para la educación y consejería a las mujeres no han sido las idóneas. Si bien la mayoría de las mujeres recibió información sobre el CaMa y $\mathrm{CaCu}$ y su detección temprana, esta se les presenta atomizada y no está integrada a su contexto, sus características y necesidades particulares. Se emplean técnicas educativas estandarizadas y unidireccionales, como las sesiones informativas (pláticas) e información impresa que resultan insuficientes e ineficientes para desarrollar conciencia real del riesgo en las mujeres.

Resulta indispensable que las actividades de educación efectiva dirigidas a la población en riesgo retomen elementos propios del contexto, que les permita a las mujeres recibir una educación en salud más cercana a sus necesidades y patrones culturales.

Por lo que se precisa adaptar los contenidos y formas de difusión de la información a las características de las mujeres y reforzarlos mediante procesos interactivos y participativos, en los que prevalezca el diálogo, la colaboración entre pares y se procure la comprensión de la información por parte de la mujer, a fin de que sean capaces de integrarla a sus prácticas cotidianas.

La educación para la salud precisa estar encaminada a brindar a la población usuaria, los conocimientos que le otorguen la capacidad de decisión y acción, desmitificando problemas de salud, apartando ideas fatalistas e infundadas, y empoderándolas en torno a su capacidad para mantener su salud, bajo el término de autonomía responsable. Para lograrlo, es indispensable que las mujeres puedan tener claro e interiorizar como una necesidad los beneficios que traen consigo las prácticas de prevención y detección precoz. Resulta relevante también evaluar los conocimientos del personal de salud, quienes son los responsables de brindar la consejería y educar a las mujeres, pues hay evidencias de que la falta de consenso entre los proveedores de salud pudiera confundir a las mujeres.

Sin duda, los programas y normas en el nivel formal (discursivo) son en su mayoría congruentes con las necesidades de la población femenina en riesgo, no obstante, en su nivel operativo no se ejercen tal como están planteadas, principalmente porque prevalece en todo el sistema de salud en México el enfoque curativo a la enfermedad y no del cuidado de la salud. Se mantiene una respuesta reactiva a la demanda de atención de la población enferma, a la cual se suman los limitados recursos asignados al primer nivel de atención, además del uso de exiguas técnicas de comunicación y educación en salud, una falta de organización y coordinación en las entidades prestadoras de servicios de salud. Todo ello propicia la duplicidad de servicios en mujeres que acuden a la atención pública y de la seguridad social, así como un marcado desinterés por parte de la población.

La accesibilidad a los servicios es un determinante importante para una detección precoz, la excesiva demanda de atención, las limitaciones de recursos humanos y materiales en los servicios públicos incrementan los tiempos de espera y pueden desmotivar a las mujeres para acudir a realizarse una valoración clínica o el tamizaje. Constituyéndose tales limitantes en barreras institucionales de acceso para que las mujeres se acerquen y hagan uso de los servicios de detección oportuna de manera regular.

\section{CONCLUSIONES}

El panorama que enfrenta México a corto y mediano plazo en cuanto al CaMa y $\mathrm{CaCu}$ no se vislumbra alentador. La creación y puesta en marcha de los programas de prevención y detección oportuna ha resultado insuficiente para refrenar la aparición de casos y muertes por estas enfermedades.

Se deduce indispensable efectuar ajustes que aumenten la probabilidad de éxito de los programas, tales como: la capacitación del personal de salud con estricto apego a la normativa y el establecimiento de un equipo de trabajo permanente para estas acciones en las unidades de salud, además de promover en el personal de salud la consciencia y sensibilidad ante esta problemática a fin de que se muestre abierto, motivado y dispuesto a educar y guiar a la población con una base de conocimientos homogéneos y la comprensión de los determinantes culturales y del 
contexto para que las mujeres sean conscientes de su riesgo.

También es necesario dirigir una mayor atención a grupos que son identificados como vulnerables, por la relevancia de los factores de riesgo asociados. Revisar y reajustar las estrategias utilizadas para difundir los conocimientos de salud, así como desarrollar herramientas de evaluación que permitan corroborar que la información ha sido comprendida y en su defecto, si no fue así, replantearla de manera que derive en un verdadero aprendizaje.

Es factible superar las barreras de acceso de muchas mujeres a los servicios de salud mediante la culturización hacia el autocuidado, trabajo nada sencillo que implica una vasta coordinación entre las instituciones de salud, así como la redistribución de recursos que le abonen significativamente a las acciones preventivas y de diagnóstico oportuno más que a las curativas.

\section{BIBLIOGRAFÍA}

Agudelo, M. (2013). Determinantes sociodemográficos del acceso a la detección del cáncer de mama en México: una revisión de las encuestas nacionales. Salud Colectiva; 9(1):79-90.

Aranguren Pulido LV, Burbano Castro JH, González JD, Mojica Cachope AM, Plazas Veloza DJ, Prieto Bocanegra BM. (2017). Barreras para la prevención y detección temprana de cáncer de cuello uterino: revisión. Investigación en Enfermería Imagen yDesarrollo.;19(2):129-43. Disponible en: http://dx.doi.org/10.11144/Javeriana.ie19-2.bpdt

Campero, L., Atienzo, EE., Marín, E., De la VaraSalazar, E., Pelcastre-Villafuerte, B., González, G (2014). Detección temprana de cáncer de mama y cervicouterino en localidades con concentración de población indígena en Morelos. Salud Pública de México. 56:511-518.

Chávarri-Guerra, Y., Villarreal-Garza, C., Liedke, P., Knaul, F., Mohar, A., Finkelstein, D., et al. (2012). Breast cancer in Mexico: a growing challenge to health and the health system. The Lancet Oncology.13: 335- 343.

Córdova-Cadena, S., González-Pozos, PV,. ZavalaGonzález, MA. (2015). Autoexploración mamaria en usuarias del Instituto Mexicano del Seguro Social de Teapa, Tabasco, México. Revista Médica del Instituto Mexicano del Seguro Social. 53(3):368-72.
Ehrenzweig, Y., Marván, ML., Acosta, EA. (2013). Conocimientos sobre la prevención del cancer cervicouterino, locus de control y realización del Papanicolaou. Psicología y Salud; 23(2):161-169.

Encuesta Nacional de Salud. Resultados Nacionales 2012. Instituto Nacional de Salud Pública. Cuernavaca.

Gallegos, I.E., Zonana, A., Gonzáles, L.E. \& Zapién, H.K. (2014). Realización de la autoexploración mamaria posterior a la atención preventiva integrada en mujeres adscritas a una Unidad de Medicina de Familia. Revista Iberoamericana de Educación e Investigación en Enfermería;4(4):49-54.

Gutierrez, J., Rivera, J., Shama, T., Oropeza, C., Ávila, MH. (2012). Encuesta Nacional de Salud y Nutrición 2012. Resultados nacionales Cuernavaca, México: Instituto Nacional de Salud Pública. pp 93,94. [Citado 01 de julio de 2016]. Disponible en: http://ensanut.insp.mx/informes/ENSANUT2012Resu $\underline{\text { ltadosNacionales.pdf }}$

Instituto Nacional de Estadística y Geografía. Estadísticas a propósito de Día mundial contra el cáncer (2014). [Internet]. [Citado 27 de agosto de 2016]. Disponible en:

http://www.inegi.org.mx/inegi/contenidos/espanol/pre nsa/Contenidos/estadisticas/2014/cancer24.pdf

Jacobo-Galindo, T., Villalobos-Arámbula, L., GallegosTorres, R., Xeque-Morales, AS., Reyes-Rocha, B., Palomé-Vega, G. (2014). Patrones de comportamiento para la detección de CA mamario en trabajadoras de una institución hospitalaria. Enfermería Universitaria. 11(3):101-109.

Knaul FM, Bhadelia A, Gralow J, Arreola-Ornelas H, Langer A, Frenk J. (2012). Meeting the emerging challenge of breast and cervical cancer in low-and middle-income countries. International Journal of Gynaecology \& Obstetrics;119 (Suppl 1): S85-8. Epub 2012 Aug 9. PubMed: 2012;11985-8..11985-8.

Knaul, M., Arreola-Ornelas H. (2014). Numeralia sobre Cáncer de cérvix y mama en México. Basado en datos de la Dirección General de Información en Salud (DGIS). Base de datos de defunciones generales 19792012. [Citado 27 de agosto de 2016]. Disponible en: http://www.tomateloapecho.org.mx/Pdfs/Numeralias/ 2014/CAMA-CACU2014.pdf

Llanes, A., Torres, I., Gómez, C. Ochoa D. (2011). El cáncer cérvico uterino, enemigo número uno de la mujer. Revista electrónica Medicina, Salud y Sociedad. 1(3). [Citado 12 de julio de 2016]. Disponible 
http://cienciasdelasaluduv.com/revistas/index.php/mss /article/view/31

López-Carrillo, L., Torres-Sánchez, L., Blanco-Muñoz, J., Hernández-Ramírez, RU., Knaul, FM. (2014). Utilización correcta de las técnicas de detección de cáncer de mama en mujeres mexicanas. Salud pública de México.; 56(5):538-46.

López-Guevara, SJ., Castillo-Muraira, Y., Ávila-Medina, M., Cano-Fajardo, LE., Ponce-Martínez, D., QuevedoDíaz, MM. (2014). Autocuidado en mujeres en relación a la prevención del cáncer de mama. Revista Paraninfo Digital. 20. [Citado 01 de julio de 2016].

Disponible en: f.com/para/n20/246.php http://www.index-

Norma Oficial Mexicana para la prevención, diagnóstico, tratamiento, control y vigilancia epidemiológica del cáncer de mama. NOM-041-SSA2-2011 (2011). México, DF Diario oficial de la Federación.

Norma Oficial Mexicana NOM-014-SSA2-1994, Para la prevención, detección, diagnóstico, tratamiento, control y vigilancia epidemiológica del cáncer cérvico uterino (2007) México, DF. Diario Oficial de la Federación. [internet]. [Citado 2 de julio de 2016]. Disponible en: http://www.salud.gob.mx/unidades/cdi/nom/m014ssa 294.pd

Organización Mundial de la Salud. Cáncer. Nota descriptiva $N^{\circ} 297$. [Internet] Actualizado en Febrero de 2013. [ Citado 15 de junio de 2014]. Disponible en: http://www.who.int/mediacentre/factsheets/fs297/es/

Pérez, SE., Romero, AL., Benítez, LA., Sandoval, L. (2011). Conocimientos y comportamientos sobre factores de riesgo de cáncer de mama en un grupo de mujeres. Revista Enfermería Universitaria ENEOUNAM. 8(1):26-32.

Plan Nacional de Desarrollo 2013-2018 Gobierno de la Republica. Mexico (2013). Disponible en: https://transparencia.info.jalisco.gob.mx/sites/default/ files/PLAN_NACIONAL_DE_DESARROLLO_2013 -2018 0.pdf

Programa de Acción Específico. (2014). Prevención y control del cáncer de la mujer 2013-2018. México, Secretaría de Salud. Disponible en: https://www.gob.mx/salud/acciones-yprogramas/programa-de-accion-especificoprevencion-y-control-del-cancer-de-la-mujer-2013$\underline{2018-9275}$
Sánchez, J.J. (2012). Tendencias de mortalidad por cáncer cervicouterino en las siete regiones socioeconómicas y en las 32 entidades federativas de México en los años 2000-2008. Gaceta Médica de México, 148:4251.Disponible http://www.medigraphic.com/pdfs/gaceta/gm2012/gm121g.pdf

Sánchez, Y., Urdaneta, JR., Villalobos, N., Contreras, A., García, J., Baabel, N., ... Cepeda, M. (2016) Conocimiento sobre cáncer de mamas y práctica del autoexamen de mamas en mujeres de edad mediana. Revista Venezolana de Oncología;28(1):37-51.

Secretaría de Salud. Información estadística. Estadísticas de Cáncer de Mama y Cáncer Cérvico Uterino (2015). [internet]. [Citado 15 de julio de 2016]. Disponible en: http://www.gob.mx/salud/acciones-yprogramas/informacion-estadistica

Siegel R, Nishadham D, Jemal A. (2012) Cancer satistics for Hispanics/Latinos, (2012). CA A Cancer Journal for clinicians; 62(5):283-298. doi: $10.3322 /$ caac. 21153

Smith, R., Manassaran, D., Brooks, D., Doroshenk, M., Fedewa, S., Saslow, D., Brawley, O., Wender R. (2015). Cancer Screening in the United States, (2015): A Review of current American Cancer Society guidelines and current Issues in cancer screening. $C A$ : A Cancer Journal for Clinicians; 65(1): 30-54.

Tejada-Tayabas, LM., Amell, L., Monarrez-Espino, J. (2015). Medical therapeutic itineraries of women with breast cancer diagnosis affiliated to the people's health insurance in San Luis Potosí, central Mexico. Cadernos de Saúde Pública; 31(1): 60-70.

Urdaneta, J.R., Nava, M.L., García, J., Cepeda, M., Zambrano, NB., Salazar, J., ... Mujica, A. (2013). Conocimiento del Cáncer de Cuello Uterino y Hallazgos Citológicos en Mujeres de Estratos Socioeconómicos Bajos. Revista Venezolana de Oncología, 25(4), 211-228. Disponible en: http://www.redalyc.org/articulo.oa?id=375634881003

Vargas-Hernández, V.M, Vargas-Aguilar, V.M. \& TovarRodríguez, J.M. (2015). Detección primaria del cáncer cervicouterino. Cirugía y Cirujanos; 83(5):448-453.

World Health Organization. GLOBOCAN (2012). Estimated cancer incidence, mortality and prevalence worldwide in 2012. International Agency for Research on Cancer [Internet]. Disponible en: http://globocan.iarc.fr/ 
Promoción de la salud, detección precoz de cáncer,

World Health Organization. GLOBOCAN (2012). Breast Cancer Estimated Incidence, Mortality and Prevalence Worldwide in 2012. México Breast 2016. [Internet1. [Citado 19 de junio de 20161. Disponible en: http://globocan.iarc.fr/old/burden.asp?selection_pop= 126484\&Text-

$\mathrm{p}=$ Mexico\&selection_cancer $=3152 \&$ Text-

$\mathrm{c}=$ Breast \&pYear $=13 \&$ type $=1 \&$ window $=1 \&$ submit $=$ $\% \mathrm{C} 2 \% \mathrm{~A} 0$ Execute

Yépez-Ramírez, D., De la Rosa, AG., GuerreroAlbarrán, C., Gómez-Martínez, JM. (2012). Autoexploración mamaria: conocimiento y perspectiva en mujeres. Revista de Enfermería del Instituto Mexicano del Seguro Social. 20(2):79-84. 